\title{
Controlling the transverse instability of dark solitons and nucleation of vortices by a potential barrier
}

\author{
Manjun Ma, ${ }^{1}$ R. Carretero-González, ${ }^{2}$ P. G. Kevrekidis, ${ }^{3}$ D. J. Frantzeskakis, ${ }^{4}$ and B. A. Malomed ${ }^{5}$ \\ ${ }^{1}$ College of Science, China Jiliang University, Hangzhou, Zhejiang, 310018, P.R. China \\ ${ }^{2}$ Nonlinear Physics Group, Departamento de Física Aplicada I, \\ Universidad de Sevilla, Avda. Reina Mercedes s/n., 41012 Sevilla, Spain \\ ${ }^{3}$ Department of Mathematics and Statistics, University of Massachusetts, Amherst MA 01003-4515 \\ ${ }^{4}$ Department of Physics, University of Athens, Panepistimiopolis, Zografos, Athens 15784, Greece \\ ${ }^{5}$ Department of Physical Electronics, School of Electrical Engineering, \\ Faculty of Engineering, Tel Aviv University, Tel Aviv 69978, Israel
}

(Dated: May 3, 2022)

\begin{abstract}
We study possibilities to suppress the transverse modulational instability (MI) of dark-soliton stripes in two-dimensional (2D) Bose-Einstein condensates (BECs) and self-defocusing bulk optical waveguides by means of quasi-1D structures. Adding an external repulsive barrier potential (which can be induced in BEC by a laser sheet, or by an embedded plate in optics), we demonstrate that it is possible to reduce the MI wavenumber band, and even render the dark-soliton stripe completely stable. Using this method, we demonstrate the control of the number of vortex pairs nucleated by each spatial period of the modulational perturbation. By means of the perturbation theory, we predict the number of the nucleated vortices per unit length. The analytical results are corroborated by the numerical computation of eigenmodes of small perturbations, as well as by direct simulations of the underlying Gross-Pitaevskii/nonlinear Schrödinger equation.
\end{abstract}

\section{INTRODUCTION}

Since the experimental creation of atomic BoseEinstein condensates (BECs) [1], a great deal of experimental and theoretical efforts has been invested into studies of nonlinear coherent matter waves. The intrinsic nonlinear nature of BECs, originating from the interatomic interactions (accounted for by an effective mean field), together with the techniques available for the generation of diverse initial configurations in the condensates, and the tunability of both external trapping potentials and intrinsic nonlinearity, have made it possible to study an extremely rich variety of nonlinear macroscopic excitations [2, 3]. In this context, it is relevant to stress that the effective dimensionality may be chosen as corresponding to the underlying three-dimensional (3D) geometry, or reduced to nearly 2D (pancake-shaped) and 1D (cigar-shaped) settings, by applying a strong confinement in the "undesirable" direction(s) 2]. On the other hand, the $s$-wave scattering length, which determines the nonlinearity strength in the framework of the mean-field description of the BEC, may be manipulated by dint of the magnetically- [4], optically- [5] or confinement- [a] induced Feshbach resonances. In combination with the use of properly designed trapping (magnetic and/or optical) potentials, such as optical lattices [7], this technique paves the way towards a versatile and exceptionally accurate control over the nonlinear matter waves. Among so generated nonlinear modes, matter-wave solitons have been observed in a series of famous experiments. In particular, nearly-1D bright and dark solitons were created in BECs with, respectively, attractive [8 10] and repulsive [11 17] interatomic interactions. Bright solitons of the gap type have also been created in the quasi-1D repulsive BEC [18] loaded into an optical lattice.
The description of the dynamics of matter waves is based on the Gross-Pitaevskii equation (GPE) 2], which bears significant similarities to the nonlinear Schrödinger equation (NLSE) governing the transmission of light signals in nonlinear optical media. Accordingly, the matterwave solitons are counterparts of optical solitons, that have been studied in detail, in temporal, spatial [19] and spatio-temporal [20] settings alike.

In this work, we focus on dark solitons (see the reviews [21, 22] for optical and matter-wave dark solitons, respectively), in connection to higher-dimensional geometry. In that case, an important issue is the stability of dark matter waves, which was first analyzed in the framework of the (2+1)-dimensional NLSE. In particular, the stability of the dark-soliton stripe (DSS) was studied in Ref. 23] (see also Refs. 24, 25]), where it was shown that the DSS is prone to the transverse modulational instability (MI) (alias "snaking instability") against transverse long-wavelength perturbations. Experimental [26, 27] and theoretical 21, 28] studies of this instability in the context of nonlinear optics have revealed that it may lead to splitting of the DSS into a chain of vortices with alternating topological charges (vortex-anti-vortex pairs). In particular, it was found that a quiescent ("black") DSS is vulnerable to transverse "snaking" deformations [21, 28], causing the splitting into vortex pairs. Unstable moving ("gray") DSSs do not split into vortices, but rather emit radiation in the form of sound waves. In the BEC context, the transverse MI and splitting of DSSs into vortex rings was first observed in the experiment with a twocomponent BEC composed of ${ }^{87} \mathrm{Rb}$ atoms in two different hyperfine states [29]. In that work, a DSS was created in one component, and the snaking instability caused it to decay into vortex rings (in a quasi-spherical geometry), in accordance with the theoretical predictions [30]. 
Several works proposed possibilities of suppressing the snaking instability of DSSs. In particular, in the context of nonlinear optics it is known 21] that this instability can be avoided in finite-size holding optical beams 31. Similarly, in the BEC context, it was shown 32] (see also Ref. [33]) that the snaking instability may be suppressed in sufficiently strong traps. On the other hand, it has been shown that 2D DSS can be stabilized by nonlocal nonlinearities 34]. Furthermore, 3D dark solitons 35] may be stabilized in dipolar condensates, which are characterized by long-range dipole-dipole interactions (see a recent review of this topic in Ref. [36]). In that case, the suppression of the DSS instability is provided by the nonlocal character of the respective mean-field model (see also Ref. 37] for similar results in the context of optics). A more complex dark-soliton configuration, which is not subject to the MI, was reported in Refs. 38, 39] in the context of the two-component BEC: it features a "cross" formed by the intersection of two rectilinear domain walls, with the wave functions of the same species filling each pair of opposite quadrants, with a $\pi$ phase difference between them. In this way, a quasi-dark-soliton configuration is formed, which is stable for long times (even in the rotating trap) in a large parametric region.

In the present work, we propose and analyze an experimentally relevant scheme for suppressing and controlling the transverse instability of DSSs in BECs and optics, based on the use of a repulsive quasi-1D potential barrier, which can be generated by a blue-detuned laser beam in BECs (see, e.g., Ref. [40]), or by a slab with a lower value of the refractive index embedded into the self-defocusing bulk waveguide. This barrier, which repels the atoms in the condensate or the light in the waveguide, creates a channel where a DSS can be naturally trapped. Analyzing the transverse MI of the DSS in this setting, we show that the channel can suppress the snaking instability. In fact, results produced by our linear-stability analysis and corroborated by direct simulations indicate that the wave number of the most unstable perturbation mode is shifted due to the presence of the potential barrier. The control of the most unstable mode is useful not only for the complete stabilization of the DSS, but also for controlling the number of vortex pairs generated by the snaking instability. This control mechanism can be adjusted by appropriately tuning the height and transverse width of the potential barrier.

The paper is organized as follows. In Sec. II, we introduce the model and produce an approximate solution for the DSS, in the presence of the potential barrier. Using the variational approximation, we obtain a solution for the DSS and analytically predict the critical wavenumber for the MI modes, by means of the linear-stability analysis (details for the latter are described in the appendix). In Sec. III] we numerically study the transverse MI of the DSS, varying the height and width of the stabilizing potential barrier. We find that, in the uniform space (i.e., in the absence of an external harmonic trapping potential) and with a sufficiently broad repul- sive potential barrier, there exists a critical value of its strength (height), above which DSSs get completely stabilized against the MI. In the case when the instability is not completely suppressed, we explore the possibility of controlling the density of the nucleated vortex pairs for an infinitely long DSS, by tuning the most unstable mode, via the parameters of the barrier. We then consider the more realistic model of a harmonically confined BEC (which may be relevant to optics too, representing a rod-shaped bulk waveguide), and perform numerical simulations which demonstrate the degree of the control over the number of the nucleated vortex pairs. In Sec.IV. we summarize the findings and point out directions for future work.

\section{THE MODEL AND ITS ANALYTICAL CONSIDERATION}

\section{A. The fundamental equation and dark-soliton solution}

We start by considering the following $(2+1)$ dimensional GPE/NLSE in the usual scaled form, with the repulsive nonlinearity:

$$
i \frac{\partial u}{\partial t}=-\frac{1}{2}\left(\frac{\partial^{2} u}{\partial x^{2}}+\frac{\partial^{2} u}{\partial y^{2}}\right)+|u|^{2} u+V(x, y) u .
$$

In the BEC context, this equation governs the evolution of the macroscopic wave function $u(x, y, t)$ of the pancake-shaped condensate in the $(x, y)$ plane [2, 3]. The external potential is assumed to have the following form:

$$
V(x, y)=V_{\mathrm{HT}}(x, y)+V_{\mathrm{LS}}(x),
$$

that includes an external harmonic trap with frequencies $\omega_{x, y}$,

$$
V_{\mathrm{HT}}(x, y)=\frac{1}{2}\left(\omega_{x}^{2} x^{2}+\omega_{y}^{2} y^{2}\right),
$$

and the potential barrier (corresponding to the fardetuned laser sheet illuminating the BEC) with the Gaussian profile:

$$
V_{\mathrm{LS}}(x)=A \exp \left[-x^{2} /\left(2 \sigma^{2}\right)\right] .
$$

Here $A$ and $\sigma$ measure the height and width of the barrier, with $A>0(A<0)$ corresponding to the blue- (red-) detuned laser beam, which repels (attracts) atoms in the condensate (note that $A<0$ corresponds to a potential trough of depth $|A|$, rather than a barrier).

In terms of nonlinear optics, Eq. (11) governs the evolution of the local amplitude of the electromagnetic wave in the self-defocusing bulk waveguide, with $t$ replaced by the propagation distance $z$, while $x, y$ are the transverse coordinates, and $V(x, y)$ describes a local modulation of the refractive index. The barrier potential (4) then corresponds to a slab with a lower value of the refractive 


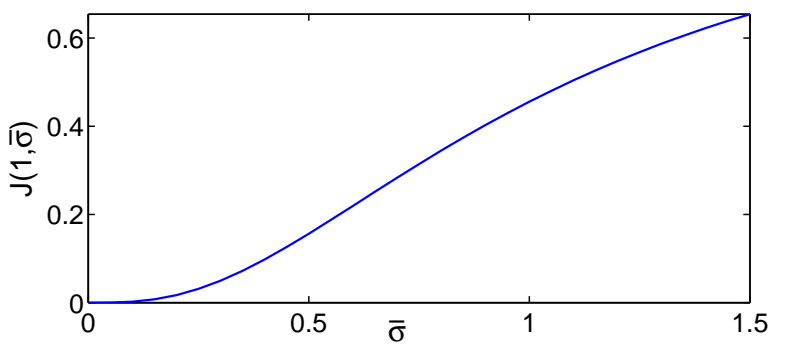

FIG. 1: (Color online) Integral (12) as a function of the rescaled width $\bar{\sigma}=\beta \sigma$.

index embedded into the waveguide, while potential (3) approximates for the global waveguiding structure.

In the absence of the external potential, $V(x, y)=0$, Eq. (11) admits an exact analytical solution for the DSS. Assuming that its nodal plane is oriented along the $x$ direction, to be aligned with the potential barrier, when it is switched on, the DSS solution is

$$
u(x, y, t)=\sqrt{\mu}\{\mathcal{B} \tanh [\sqrt{\mu} \mathcal{B}(x-v t)]+i \mathcal{A}\} e^{-i \mu t} .
$$

Here, $\sqrt{\mu} \exp (-i \mu t)$ is the stationary background with density $\mu$ (which is actually equal to the normalized chemical potential) supporting the dark soliton. Further, $\sqrt{\mu} \mathcal{B}$ and $v=\sqrt{\mu} \mathcal{A}$ represent, respectively, the soliton's depth (or inverse width) and velocity, with $\mathcal{A}$ and $\mathcal{B}$ subject to the constraint $\mathcal{A}^{2}+\mathcal{B}^{2}=1$. The DSS with $\mathcal{A}=0$ $(\mathcal{A} \neq 0)$ is stationary (moving), and is usually called a "black" ("grey") dark soliton. Below, we first employ the variational approximation to find an approximate stationary DSS solution to Eq. (10) in the presence of the potential barrier (4), and then perform the linear-stability analysis to study the transverse MI.

\section{B. Dark-soliton stripe steady state in the presence of the potential barrier.}

In the absence of the harmonic trapping potential (3), but in the presence of barrier (44), the profile of a stationary DSS solution to Eq. (1) is sought for as

$$
u(x, y, t)=U(x) e^{-i \mu t},
$$

where $\mu$ is the chemical potential (or $-\mu$ is the propagation constant, in terms of the optical model), and $U(x)$ is a real function. Substituting this into Eq. (1) leads to an ordinary differential equation,

$$
\frac{1}{2} U^{\prime \prime}+\left[\mu-A e^{-x^{2} /\left(2 \sigma^{2}\right)}\right] U-U^{3}=0 .
$$

As Eq. (7) describes both the dark soliton and the uniform background, one should subtract the contribution of the latter into the respective Lagrangian, which will be used as the basis of the variational approximation. To this end, we follow Ref. [41] (see also Ref. [42]), and write the Lagrangian as follows,

$$
L=\left(U^{\prime}\right)^{2}-2\left[\mu-A e^{-x^{2} /\left(2 \sigma^{2}\right)}\right]\left(U^{2}-\mu\right)+\left(U^{4}-\mu^{2}\right),
$$

where $\mu$ is the amplitude of the uniform background, as defined above. Then, to approximate the stationary dark-soliton solution of Eq. (77) we use the following ansatz [cf. Eq. (5)]

$$
U(x ; A, \sigma)=\sqrt{\mu} \tanh (\beta(A, \sigma) x),
$$

where the inverse width of the soliton, $\beta$, is a variational parameter, while the density $\mu$ is the fixed background chemical potential. Inserting ansatz (9) into the Lagrangian (8) and performing the integration over $-\infty<x<+\infty$, we arrive at the effective (averaged) Lagrangian:

$$
L_{\mathrm{eff}}=\frac{4}{3} \mu\left(\beta+\frac{\mu}{\beta}\right)-2 A \mu \int_{-\infty}^{+\infty} e^{-x^{2} /\left(2 \sigma^{2}\right)} \operatorname{sech}^{2}(\beta x) d x .
$$

Further, we use the Euler-Lagrange equation, $\partial L_{\text {eff }} / \partial \beta=$ 0 , to derive the following implicit equation for the inverse soliton's width $\beta$ :

$$
\beta^{2}=\mu-3 A \beta^{2} J(\beta, \sigma),
$$

where

$$
J(\beta, \sigma) \equiv \int_{-\infty}^{+\infty} x e^{-x^{2} /\left(2 \sigma^{2}\right)} \operatorname{sech}^{2}(\beta x) \tanh (\beta x) d x .
$$

Using the rescaling, $x \rightarrow \beta x$, we conclude that $J(\beta, \sigma)=$ $J(1, \bar{\sigma}) / \beta^{2}$, where $\bar{\sigma} \equiv \beta \sigma$, hence one can rewrite Eq. (11) as

$$
\beta^{2}=\mu-3 A J(1, \beta \sigma) .
$$

Apparently, Eq. (13), with $J$ being a function of the single argument, which is depicted in Fig. 1 versus rescaled width $\bar{\sigma}$, is simpler than Eq. (11).

In Fig. 2 we validate the results of the variational approximation by comparing the width extracted from the numerically found steady state, and from the variational equation (13) [thick (blue) and thin (red) lines, respectively], for different values of the barrier's parameters. As seen in the figure, for $A>0$ (i.e., for the repulsive potential barrier), the variational approximation is very accurate, with the steady-state solution closely resembling the tanh profile of ansatz (9) [see panel (b) in the figure]. On the other hand, it is seen in panel (c) that for $A<0$ (the attractive potential trough, instead of the barrier) the approximation deteriorates, especially for large widths of the trough $(\sigma>0.6$, which exceeds the width of the DSS). This observation is explained by the fact that the steady-state profile develops a localized hump induced by the attractive trough (see also Ref. [43]), which is not captured by our ansatz. 

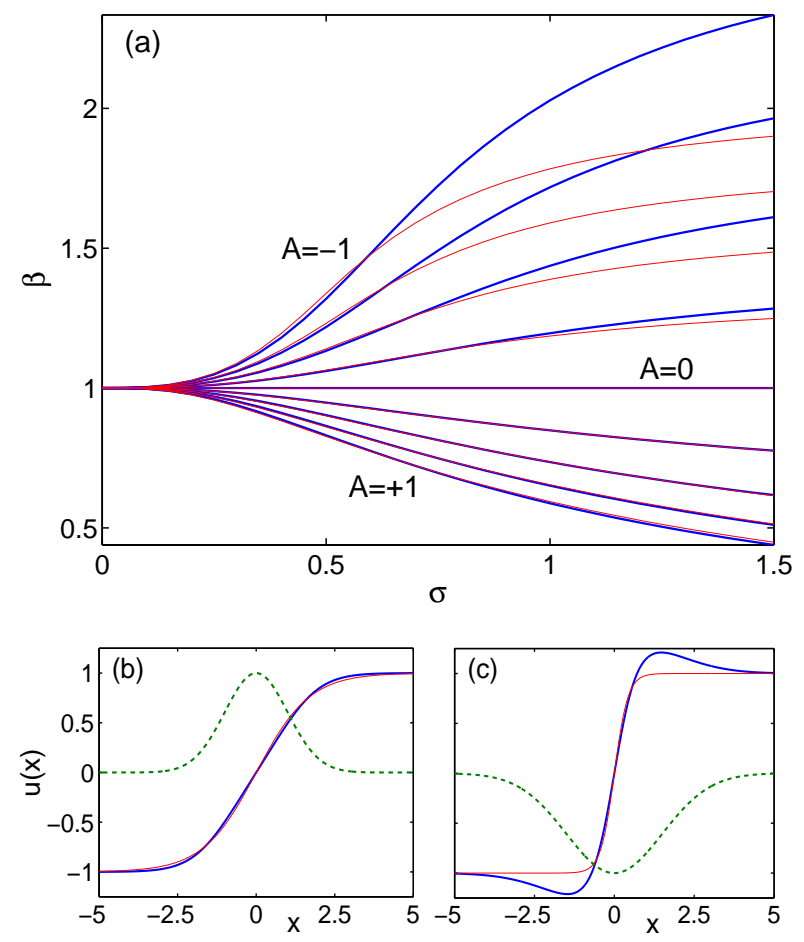

FIG. 2: (Color online) (a) Inverse width $\beta$ of the dark soliton as a function of width $\sigma$ of the potential barrier, with height $A$ ranging from $A=-1$ (top curves) to $A=+1$ (bottom curves) in steps of 0.25 . The thick (blue) curves depict the width of the numerical solution, obtained by fitting its profile to $\tanh (\beta x)$. The thin (red) lines represent the results predicted by the variational approximation, see Eq. (13). Panels (b) and (c) depict, respectively, two solutions for $(A, \sigma)=(1,1)$ and $(A, \sigma)=(-1,1.5)$, the latter case corresponding to the potential trough, rather than a barrier. The thick (blue) line depicts the numerically found steady state, while the thin (red) line represents the fitted $\tanh (\beta x)$ profile. The transverse profile of the potential barrier is depicted by the dashed (green) line. The chemical potential is fixed to be $\mu=1$.

\section{The transverse modulational instability of the dark-soliton stripe in the presence of the potential barrier.}

We now consider the solution of Eq. (1) in the form of the DSS in the presence of the potential barrier. In this case, the steady state DSS may be approximated by

$$
u(x, y, t)=u_{0}(x, y) e^{-i \mu t}=\sqrt{\mu} \tanh (\beta x) e^{-i \mu t},
$$

where $\beta$ is determined by Eq. (13). To analyze the MI of this DSS, we follow the approach of Ref. [23] and consider small transverse perturbations, see details in the appendix. The result of the analysis presented in the appendix is that the critical wavenumber which defines the width of the instability band for solution (6) is

$$
k_{\mathrm{cr}}=\beta,
$$

where $\beta$ is determined by Eq. (13). That is, the DSS (14) is unstable against transverse perturbations with
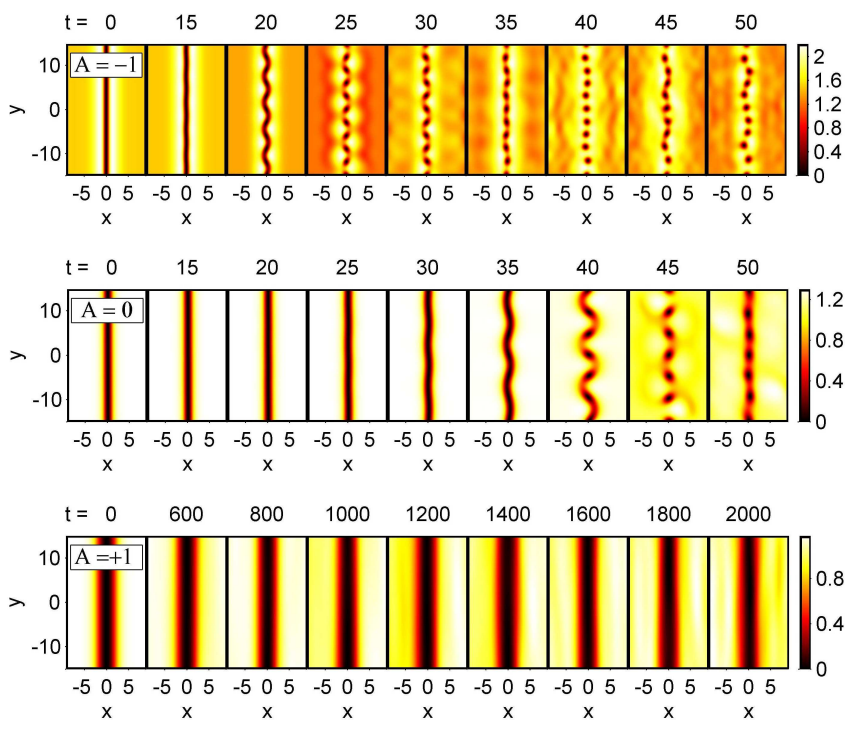

FIG. 3: (Color online) The dynamics induced by the transverse modulational instability of a dark-soliton stripe in the absence of the external harmonic trap. Shown are different density snapshots at indicated moments of time. The initial condition corresponds to a stationary stripe with an added random perturbation of relative size $10^{-5}$. The top panel: the case of the attractive potential trough, with $A=-1$ and $\sigma=1.5$. The middle panel: no potential barrier or trough $(A=0)$. The bottom panel: the case of the repulsive potential barrier, with $A=1$ and $\sigma=1.5$. Note the faster destabilization and nucleation of a larger number of vortices (see dark circular spots for later times) in the case of the attractive potential trough (the top panel), in comparison to the case when the trough is absent (the middle panel), and the complete stabilization of the dark-soliton stripe by the repulsive potential barrier in the bottom panel. The simulations in this figure were carried out in the domain of $(x, y) \in[-15,15] \times[-15,15]$.

wavenumbers $k<k_{\mathrm{cr}}=\beta$. When this instability sets in, the DSS undergoes a snake-like transverse deformation, which eventually breaks it into vortex-antivortex pairs. This outcome of the evolution can be observed in Fig. 3 .

From Eq. (15) we can derive the critical number of vortices in the chain produced by the splitting of the DSS (14):

$$
N_{\mathrm{cr}}=\beta L / \pi,
$$

where $L$ is the length of the DSS. Since $\beta$ is implicitly determined by height $A$ and width $\sigma$ of the potential barrier (4), as per Eq. (13), it is possible to control the critical wavenumber by appropriately adjusting the barrier's parameters. This, in turn, offers a control of the upper bound of the vortex pairs that are nucleated by the transverse instability of the DSS. In the next section we show that it is indeed possible to control the number of the nucleated vortex pairs, which we compare with the analytical prediction. 

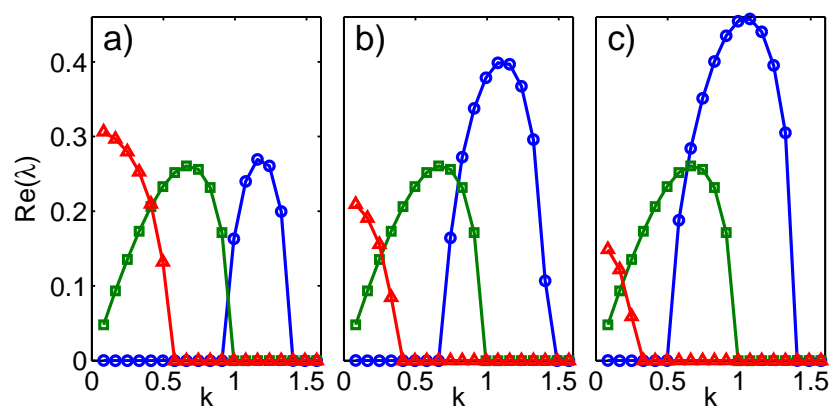

FIG. 4: (Color online) The growth rate of the transverse modulational instability of dark-soliton stripes at different values of the height and width of the potential barrier, $A$ and $\sigma$. The panels correspond to the increasing width: (a) $\sigma=0.5$, (b) $\sigma=1$, and (c) $\sigma=1.5$, as indicated by the dashed lines in Fig. 5. Each panel displays the real part of the eigenvalue, for the potential trough/barrier with heights $A=-1$ [(blue) circles], $A=0$ [(green) squares], and $A=1$ [(red) triangles]. The computations corresponding to this figure were carried out in the domain of $(x, y) \in[-50,50] \times[-50,50]$.

\section{NUMERICAL RESULTS}

A. Transverse modulational instability of the dark-soliton stripe in the absence of the harmonic trap

The main subject of this work is the control of the transverse MI of the DSS by means of the potential barrier. Typical examples of the snaking instability are depicted in Fig. 3, in the absence of the external harmonic trap $\left[\omega_{x, y}=0\right.$ in Eq. (3)]. The figure depicts the evolution of the density $|u(x, y, t)|^{2}$ at different times, starting from a stationary DSS with a small initial random perturbation added to it. The middle panel in the figure depicts the case of a quiescent (black) DSS $(\beta=1)$, with no potential barrier $A=0$, where the instability manifests itself with wavenumber $k \approx 2 \pi / 10=0.628$ (wavelength $\simeq 10$ ), which is smaller than the predicted critical wavenumber, $k_{\mathrm{cr}}=\beta=1$. In fact, this unstable wavenumber is the most unstable one, $k_{\max }$, for this configuration.

In order to quantify the instability and to compare it to the analytical results of the previous section, we compute the stability spectra of the stationary solutions $u_{0}(x, y)$ through the standard Bogoliubov-de Gennes $(\mathrm{BdG})$ analysis. This analysis involves numerically solving the linear eigenvalue BdG problem, which stems from the linearization of the GPE (1) around the steady state solution $u_{0}$ by using ansatz $u(x, y, t)=$ $\left\{u_{0}(x, y)+\left[a(x, y) e^{\lambda t}+b^{\star}(x, y) e^{\lambda^{\star} t}\right]\right\} e^{-i \mu t}$. The solution of this BdG eigenvalue problem yields the eigenfunctions $\{a(x, y), b(x, y)\}$ and the eigenvalues $\lambda$. Note that, due to the Hamiltonian nature of the system, if $\lambda$ is an eigenvalue of the BdG spectrum, so are also $-\lambda, \lambda^{*}$ and $-\lambda^{*}$. Notice that the linear stability condition amounts to $\operatorname{Re}(\lambda)=0$, i.e., all eigenvalues must be imaginary. In

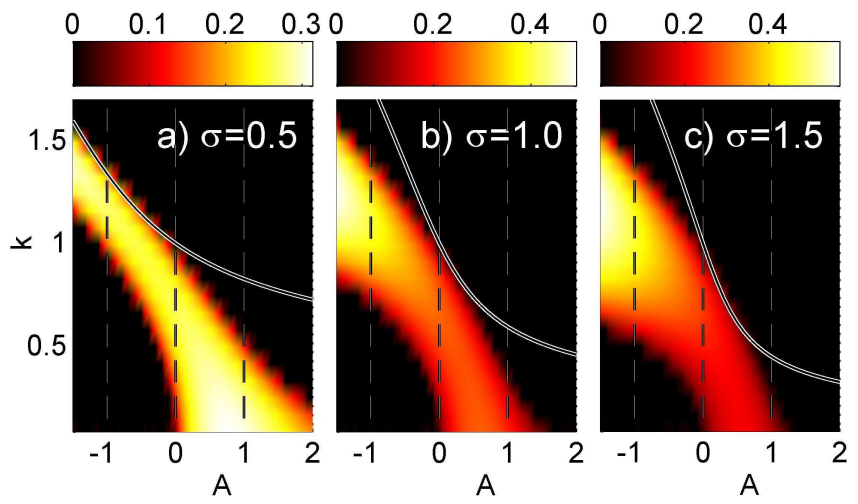

FIG. 5: (Color online) Regions of the transverse modulational instability of the dark-soliton stripe for different parameters of the potential barrier. Depicted is the real part of the eigenvalue (the lighter color represents the stronger instability, while black corresponds to the stability) as a function of wavenumber $k$ and the barrier's height $A$. Different panels correspond to increasing width of the barrier: (a) $\sigma=0.5$, (b) $\sigma=1$, and (c) $\sigma=1.5$. The black and white solid lines depict the prediction of the critical wavenumber as per Eq. (15). Vertical dashed lines represent the cuts depicted in Fig. 4

fact, in connection to the stability analysis described in the previous section, the BdG eigenvalue $\lambda$ corresponds to eigenfrequency $\Omega$ through relation $\Omega=i \lambda$. Therefore, an unstable eigenvalue corresponding to $\operatorname{Re}(\lambda)>0$ corresponds, in turn, to an eigenfrequency with $\operatorname{Im}(\Omega)>0$.

The MI spectrum for the DSS in the case of no potential barrier $(A=0)$ is depicted by the (green) squares in all panels in Fig. 4. The most unstable wavenumber, $k_{\max }$, corresponds to the location of the maxima of $\operatorname{Re}(\lambda)>0$. Note that, as predicted by the linear stability analysis, all modes with $k>k_{\mathrm{cr}}=\beta=1$ are stable. It is also interesting to note that for strong enough attractive $(A<0)$ potential barriers the whole spectrum is shifted to the right, so that small wavenumbers become stable [see (blue) circles in Fig. 4. Furthermore, it is important to note that the results presented in Fig. 4 were obtained in a large box, $(x, y) \in[-50,50] \times[-50,50]$, in order to safely capture small wavenumbers. In contrast, the simulations depicted in Fig. 3 were performed in a smaller domain box, $(x, y) \in[-15,15] \times[-15,15]$ so that the case for $A=1$ (the bottom row in the figure) is rendered stable since the corresponding unstable wavenumbers do not fit into the integration box. This effect, discussed further below, becomes important when considering trapped condensates that inherently possess a finite size.

We now turn to the effect of the potential barrier on the stability (and dynamics) of the DSS. The top panel in Fig. 3 depicts the snaking instability corresponding to the attractive potential trough with strength (depth) $A=-1$. As seen in the figure, the attractive trough naturally enhances the instability, in comparison to the free-standing DSS, in two ways: (i) the MI sets in earlier, and (ii) the most unstable wavenumber is right-shifted 
$\left(k_{\max } \approx 2 \pi / 6=1.0472\right)$. The corresponding instability spectrum for this case is depicted by the (blue) circles in Fig. 4(c). In fact, comparing the (green) squares and the (blue) dots in this panel, it is evident that the net effect of the attractive potential trough is the right-shift of the instability spectrum. Note that the spectrum is located to the left of the threshold, $k_{\mathrm{cr}}=\beta \approx 1.78$, which is predicted by the linear-stability analysis.

In contrast to the destabilizing effects of the attractive potential, the repulsive barrier stabilizes the DSS, as might be expected and is shown in the bottom panel of Fig. 3. The spectrum for this case is depicted by the (red) triangles in Fig. 4(c). Note that, although some perturbation modes are still unstable in this case, their wavelengths are too large to fit the integration domain used in the simulations shown in the bottom panel of Fig. 3 and, thus, the respective sufficiently strongly repulsive barrier renders the DSS effectively stable (in a still larger domain, we do observe the development of the weak transverse MI in this case, which is not shown here). The same stabilizing effect is also observed for other values of the widths of the barrier, as it can be clearly seen in all spectra depicted in Fig. 4. This figure depicts, for three values of width $\sigma$, the shift of the MI spectrum for the attractive [(blue) circles], zero [(green) squares] and repulsive [(red) triangles] channel potentials. The figure suggests that, as mentioned above, for a sufficiently strong repulsive potential barrier, only very small wavenumbers may be unstable. Therefore, if the longitudinal size of the condensate (set by the external trap) or optical waveguide, into which the DSS is embedded, is not too large, the instability might be fully suppressed, as the unstable wavelengths would be too large to comply with the longitudinal size (see also Ref. [32] and Sec. IIIB below).

In fact, as we now demonstrate, a sufficiently strong repulsive barrier may completely suppress all instabilities, including those with very small wavenumbers. In Fig. 5 we depict the instability spectra for three different widths of the barrier widths, as a function of the sign and strength of the channel potential. The light-colored areas in the panels correspond to unstable modes, with the shading scale corresponding to the associated instability growth rate for each mode. As shown in the figure, for sufficiently wide potential channels $(\sigma \geq 1.0$, see the two right panels), there is a positive value of the barrier's height above which all wavenumbers are stable (the zero real part of the eigenvalues corresponds to the black shade in the panels). Therefore, it is possible to completely suppress the snaking instability and render the DSS stable with the appropriate choice of the potential barrier.

For example, for the domain used in Figs. 4.5 and a potential barrier width of $\sigma=1(\sigma=1.5)$ and height larger or equal to $A=1.65(A=1.35)$ the DSS is completely stable. We also plot in Fig. 5 (see solid black and white lines) the critical wavenumber (15) obtained in the analytical form in Sec. III. This approximate result (valid for $A=0$ ) captures the qualitative behavior of the critical wavenumber, but fails to give its precise location.

Another important feature of the control over the location of the most unstable mode $k_{\max }$ is that it allows one to precisely manipulate the number of nucleated vortices per unit length which emerge from the snaking instability. The instability nucleates one vortex pair per snaking wavelength [33]. This feature is clearly visible at later times in Fig. 3. For example, the destabilizing (attractive) channel potential, with $(\sigma, A)=(1.5,-1)$, enhances the outcome by producing $N_{v}=10$ vortices in the domain of length $L=30$, namely the vortex density $\rho_{v}=10 / 30=0.33 \ldots$. On the other hand, the freestanding DSS $(A=0)$ is responsible for the nucleation of $\rho_{v}=6 / 30=0.2$ vortices per unit length. Thus, one can manipulate the density of the nucleated vortices, selecting it from zero to a maximum value which is inversely proportional to the healing length of the condensate, in the case of BEC (recall that two single-charged vortices cannot coexist at distances smaller than this length).

\section{B. The modulational instability of dark-soliton stripes in the harmonic trap}

We now consider the combined effects of the external harmonic trap and the channel potential. As mentioned above, this combination can produce an effective suppression of the snaking instability if the trap's strength is such that the corresponding Thomas-Fermi (TF) radius of the BEC, $R_{\mathrm{TF}}=\sqrt{2 \mu} / \omega\left(\right.$ for $\omega_{x}=\omega_{y} \equiv \omega$ ), is smaller than the smallest wavelength of unstable perturbations. In Fig. 6 we display the development of the DSS' snaking instability and the concomitant vortex nucleation for the BEC confined in the harmonic trap of strength $\omega=0.05$. The three panels correspond, from top to bottom, to channel potentials with width $\sigma=1.5$ and strength $A=-0.8,0$, and 0.4 . It is difficult to precisely measure the number of nucleated vortices because some of them are nucleated at the rims of the configuration and are almost invisible. Therefore, we herein focus on measuring the number of nucleated vortices, $N_{v}$, inside the circle of the TF radius. It is clear that, for $A=-0.8$, at least $N_{v}=16$ vortices are nucleated, while $N_{v}=10$ for $A=0$ (without the channel potential), and only $N_{v}=5$ vortices emerge for $A=0.4$.

Figure 7 shows the number of nucleated vortices for two different values of the trapping frequency. The curve corresponding to $\omega=0.05$ has been scaled by factor $1 / 2$ for a better comparison with the one corresponding to $\omega=0.1$, since the former has the TF radius which is twice as large. In order to estimate analytically the number of nucleated vortices inside the BEC cloud, we first establish an upper bound by considering the number of nucleated vortices in a DSS of length $L=2 R_{\mathrm{TF}}$ with the threshold wavenumber $k=k_{\mathrm{cr}}$ obtained in Sec. [II. Clearly, this estimation is merely an upper bound for the number of nucleated vortices because of the following rea- 


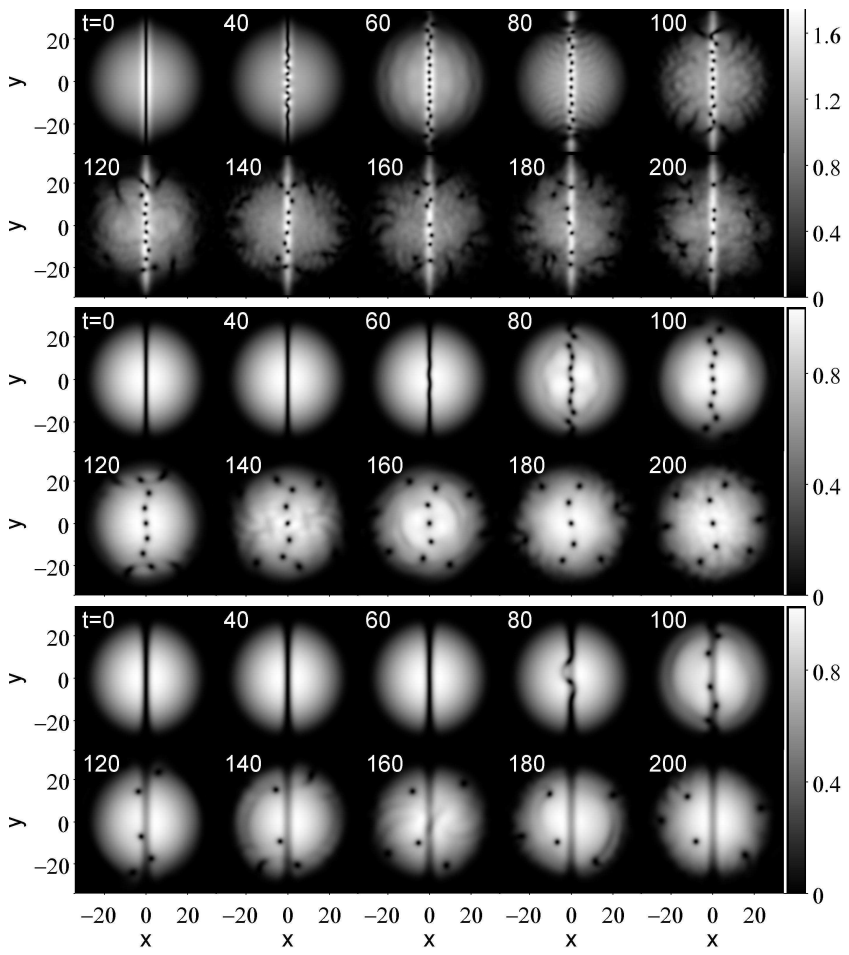

FIG. 6: Dynamics of the transverse modulational instability of a dark-soliton stripe in the BEC loaded into the harmonic trap of strength $\omega=0.05$. Shown are different snapshots of the density at the times indicated in the figure. The initial condition corresponds to a stationary stripe to which a random perturbation of relative size $10^{-5}$ was added. The top panel shows the case with the attractive potential trough of depth $A=-0.8$ and width $\sigma=1.5$. The middle panel: the situation in the absence of the channel potential $(A=0)$. The bottom panel: the case of the repulsive potential barrier of height $A=0.4$ and width $\sigma=1.5$. Note that the number of nucleated vortices can be controllably varied from about 16 for $A=-0.8$ (in the top panel) to none for $A \gtrsim 1$ (not shown here).

sons: (i) since $k_{\max }$ is not readily available from the theory, we are using $k_{\mathrm{cr}}\left(k_{\max }<k_{\mathrm{cr}}\right)$, which corresponds to the instability threshold and not to the wavenumber with the maximum growth rate; (ii) at the periphery of the TF cloud, it is difficult to pick the number of vortices through the direct count of the number of empty cores in the density profiles, and (iii) as it can be noticed from Fig. 囵, the analytical prediction for $k_{\mathrm{cr}}$ is always an overestimation of the true wavenumber threshold. Thus, an upper bound for the number of vortices can be safely given by

$$
N_{\mathrm{cr}}=\frac{\beta L}{\pi}=\frac{2 \beta R_{\mathrm{TF}}}{\pi}=\frac{2 \beta \sqrt{2 \mu}}{\pi \omega} .
$$

In connection with item (iii) above, it is worth mentioning that it is in principle possible to measure the number of vortices by analyzing the vorticity (curl of the superfluid velocity) of the condensate. This method reveals (results not shown here) that, indeed, some vortices cre-

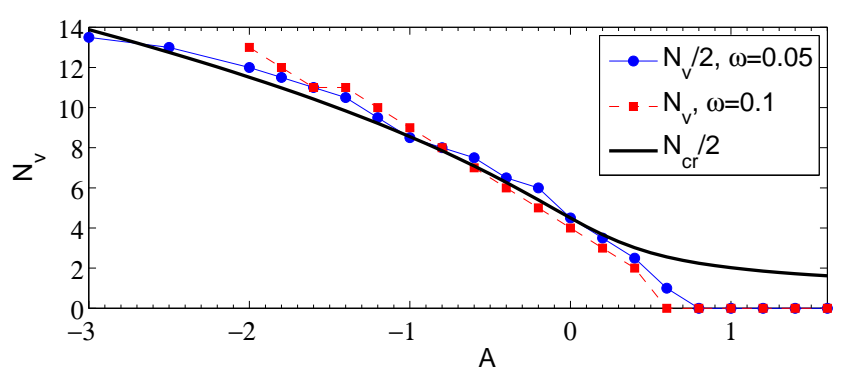

FIG. 7: (Color online) The number of nucleated vortices $\left(N_{v}\right)$ resulting from the snaking instability of the dark-soliton stripe in the BEC loaded into the harmonic trap, versus the strength $(A)$ of the channel potential. The number of vortices is measured by direct count of the number of empty cores present in the density profile after the snaking instability is fully developed. The solid line depicts half of the theoretical prediction of Eq. (17) (see text). Circles: the trap's strength corresponds to $\omega=0.05$ and depicted is the half number of the vortices $\left(N_{v} / 2\right)$. Squares: the trap's strength corresponds to $\omega=0.1$. The width of potential channel is $\sigma=1.5$ for both data sets.

ated at the periphery of the cloud are missed by the direct empty-core counting method, based on the inspection of the density profiles. Nonetheless, we have observed that these peripheral vortices are quickly pushed out and always stay at the periphery. Therefore, such boundary vortices do not induce a considerable change in the longterm BEC dynamics. It is also worth pointing out that counting vortices by the number of empty cores in the density profile is tantamount to what is often used in current BEC experiments. Of course, in order to image the vortices in real experiments, the BEC cloud has to be left to expand and this could potentially modify the density distribution and "push" vortices inwards or outwards the BEC cloud. However, since the expansion time is usually short, it is likely that any dynamics during expansion will be rather slow and, thus, no significant change in the measurable number of vortices should be expected.

As concluded above, the observable number of vortices nucleated by the DSS, $N_{v}$, will be smaller than the upper bound $N_{\text {cr }}$ defined in Eq. (17), hence a relation of the type $N_{v}=\alpha N_{\text {cr }}$, with $\alpha<1$, should be more appropriate. For the specific value of $\sigma=1.5$ used in Fig. 7 we have noticed that the most unstable growth rate $k_{\max }$ is very close to half of the critical threshold $k_{\mathrm{cr}}$ for values of the laser intensity $A<1$. Therefore, for this regime, it is sensible to choose $\alpha=0.5$ as a relevant approximation. In fact, as it can be noticed from Fig. 7, see solid black line, we have found that the choice of $\alpha=0.5$ gives a very good match to the actual number of observed vortices in the numerical experiments for a wide range of values of the potential barrier intensity $A$. It is important, however, to stress that the above estimate, relying on the assumption that $k_{\max } \approx 0.5 k_{\mathrm{cr}}$, is only valid for a wide potential barrier with $\sigma=1.5$. For other regimes, we can only give an upper bound for the number of nucle- 
ated vortices as per Eq. (17). Furthermore, it is relevant to mention that the prefactor $\alpha$ also depends (results not shown here), for other fixed values of the laser sheet's width $\sigma$, on the laser strength $A$.

\section{CONCLUSIONS}

In this work, we have proposed and analyzed a technique to control and even eliminate the transverse modulational instability, alias the snaking instability, of darksoliton stripes in Bose-Einstein condensates (BECs) and bulk optical waveguides with the self-defocusing nonlinearity. This technique relies on the use of a quasi-1D potential barrier, that may be induced by a blue-detuned laser sheet in the BEC, or by a slab embedded into the optical waveguide. We have also considered the opposite quasi-1D potential, in the form of an attractive potential trough (that may be induced by a red-detuning laser beam), which enhances the snaking instability by making the instability-onset time shorter and, more importantly, the unstable wavenumbers larger. Therefore, the attractive trough produces more instability-induced oscillations per unit length, eventually generating a larger density of vortex-antivortex pairs. Most importantly, the repulsive barrier is able to suppress the snaking-instability band, and even completely stabilize the dark-soliton stripe.

We have also developed an analytical approximation for estimating the largest unstable mode (in the absence of the external harmonic trap). The analytical results show good agreement with the numerical results obtained from the Gross-Pitaevskii equation. We also considered the model including the isotropic harmonic trap. In this case, we monitored the total number of vortices nucleated by the modulational instability inside the BEC cloud. Assuming that the size of the cloud is determined by the Thomas-Fermi radius, we were able to provide an upper bound for the estimate of the nucleated number of vortices as a function of the harmonic-trap's strength.

The stabilization mechanism proposed in this work may be naturally extended in diverse directions. First, it is interesting to consider the stabilization of quasi-1D solitons by the attractive potential trough in the model with the self-attraction, and the situation with either sign of the nonlinearity, if the channel potentials are replaced by a sufficiently strong periodic optical lattice. Another challenging possibility is to consider the stabilization of quasi-1D solitons by the cigar-shaped potential and its periodic optical-lattice counterpart in the $3 \mathrm{D}$ geometry.

\section{Acknowledgments}

R.C.G. gratefully acknowledges the hospitality of Grupo de Física No Lineal (GFNL) of Universidad de Sevilla, and support from NSF-DMS-0806762, Plan Propio de la University de Sevilla and Grant \# IAC09-I4669 of Junta de Andalucía. M. Ma gratefully acknowl- edges the hospitality of the Nonlinear Dynamical Systems (NLDS) group at the San Diego State University, where this work was carried out with the support from the state scholarship fund of P. R. China. P.G.K. gratefully acknowledges support from the NSF-CAREER program (NSF-DMS-0349023) and from NSF-DMS-0806762. The work of D.J.F. was partially supported by the Special Account for Research Grants of the University of Athens. The work of B.A.M. was, in a part, supported by the German-Israel Foundation through Grant No. 149/2006, and by a grant on the topic of "Nonlinear spatiotemporal photonics in bundled arrays of waveguides" from the High Council for Scientific and Technological Cooperation between France and Israel.

\section{Appendix: The linear stability analysis for the dark-soliton stripe}

In order to analyze the linear stability of the darksoliton stripe characterized by Eq. (14) in the presence of the potential barrier, we closely follow the approach of Ref. [23]. We consider a perturbation of the DSS in the form of

$$
\delta u=u_{1}(x) e^{\Lambda}, \quad \delta u^{*}=u_{2}(x) e^{\Lambda^{*}}
$$

with $\Lambda=-i \Omega t+i k y$, where $*$ denotes the complex conjugation, $\Omega$ is an eigenvalue in the spectrum of Eq. (1) linearized around solution (14), $\left(u_{1}, u_{2}^{*}\right)$ is the corresponding eigenfunction, and $k$ is the corresponding wavenumber along the $y$ direction.

Substituting the standard ansatz for the perturbation around the soliton solution (14),

$$
u(x, y, t)=\left(u_{0}+\delta u+\delta u^{*}\right) e^{-i \mu t}
$$

into Eq. (11), and noting the linear independence of $e^{\Lambda}$ and $e^{\Lambda^{*}}$, leads one to the following linear-stability equations:

$$
\left\{\begin{array}{r}
\frac{1}{2} \frac{\partial^{2} u_{1}}{\partial x^{2}}+\left(\mu-V-2\left|u_{0}\right|^{2}\right) u_{1} \\
-u_{0}^{2} u_{2}^{*}-\frac{1}{2} k^{2} u_{1}+\Omega u_{1}=0 \\
\frac{1}{2} \frac{\partial^{2} u_{2}^{*}}{\partial x^{2}}+\left(\mu-V-2\left|u_{0}\right|^{2}\right) u_{2}^{*} \\
-u_{0}^{* 2} u_{1}-\frac{1}{2} k^{2} u_{2}^{*}-\Omega u_{2}^{*}=0
\end{array}\right.
$$

where the original second equation has been replaced by its complex-conjugate counterpart. Equation (20) can be written in the matrix form,

$$
\Omega M \varphi-\frac{1}{2} k^{2} I \varphi+L \varphi=0,
$$

where

$$
\begin{gathered}
\varphi \equiv\left(\begin{array}{c}
u_{1} \\
u_{2}^{*}
\end{array}\right), \quad M \equiv\left(\begin{array}{cc}
1 & 0 \\
0 & -1
\end{array}\right), \quad I \equiv\left(\begin{array}{ll}
1 & 0 \\
0 & 1
\end{array}\right), \\
L \equiv \frac{1}{2} I \frac{\partial^{2}}{\partial x^{2}}-\left(\begin{array}{cc}
2\left|u_{0}\right|^{2}-\mu+V & u_{0}^{2} \\
u_{0}^{* 2} & 2\left|u_{0}\right|^{2}-\mu+V
\end{array}\right) .
\end{gathered}
$$


Rotating the eigenfunction $\varphi$,

$$
\varphi \equiv\left(\begin{array}{cc}
1 & i \\
1 & -i
\end{array}\right) \widetilde{\varphi}
$$

yields another representation of Eq. (21):

$$
\Omega\left(\begin{array}{cc}
1 & i \\
-1 & i
\end{array}\right) \widetilde{\varphi}-\frac{1}{2} k^{2}\left(\begin{array}{cc}
1 & i \\
1 & -i
\end{array}\right) \widetilde{\varphi}+L\left(\begin{array}{cc}
1 & i \\
1 & -i
\end{array}\right) \widetilde{\varphi}=0
$$

with $\widetilde{\varphi}=\left(\begin{array}{c}f_{1} \\ f_{2}\end{array}\right)$. Using the matrix $1 / 2\left(\begin{array}{cc}1 & 1 \\ -i & i\end{array}\right)$ to left multiply Eq. (23) yields

$$
\Omega M_{i} \widetilde{\varphi}-\frac{1}{2} k^{2} I \widetilde{\varphi}+\widetilde{L} \widetilde{\varphi}=0
$$

with $M_{i}=\left(\begin{array}{cc}0 & i \\ -i & 0\end{array}\right)$ and $\widetilde{L}=\frac{1}{2} I \frac{\partial^{2}}{\partial x^{2}}-\left(\begin{array}{cc}\Delta & i \Delta_{-} \\ i \Delta_{-} & \Delta^{\prime}\end{array}\right)$, where $\Delta \equiv 2\left|u_{0}\right|^{2}+\Delta_{+}-\mu+V$ and $\Delta_{ \pm} \equiv\left(u_{0}^{* 2} \pm u_{0}^{2}\right) / 2$. By using Eq. (14), $\widetilde{L}$ becomes

$$
\widetilde{L}=\frac{1}{2} I \frac{\partial^{2}}{\partial x^{2}}+\left(\begin{array}{cc}
L_{1+} & 0 \\
0 & L_{2+}
\end{array}\right)
$$

where we define

$$
L_{1+}=3 \mu \operatorname{sech}^{2}(\beta x)-2 \mu-V, L_{2+}=\mu \operatorname{sech}^{2}(\beta x)-V .
$$

As follows from Eqs. (21) and (24), the main piece of the information concerning the spectrum of eigenvalues $\Omega$ comes from properties of the operator $\widetilde{L}$. Obviously, it is a Hermitian operator, hence its spectrum is purely real. The respective eigenvalue problem can be written as

$$
\widetilde{L} \psi=\frac{1}{2} p^{2} \psi, \quad p=p^{*} .
$$

We should note that $p$ corresponds to $k$ in Eq. (24) with $\Omega=0$. Our purpose is to find the critical value $k_{\mathrm{cr}}$ of wavenumber $k$ for the instability of solution (14). To proceed, we need to look for a solution to (27) as

$$
\psi=\left(\begin{array}{c}
f_{1} \\
f_{2}
\end{array}\right)=\left(\begin{array}{c}
e^{-\beta x} g_{1}(x) \\
e^{-\beta x} g_{2}(x)
\end{array}\right),
$$

so that the perturbation functions $f_{1}$ and $f_{2}$ share the same exponential decay rate near $\pm \infty$ as that of the dark soliton $u_{0}$, where $g_{1}$ and $g_{2}$ are constants in the limit of $|x| \rightarrow \infty$, and the derivatives $d g_{i} / d x$ and $d^{2} g_{i} / d x^{2}$ are bounded in the whole real domain so that

$$
\lim _{|x| \rightarrow \infty} \frac{d g_{i}}{d x}=0, \quad \lim _{|x| \rightarrow \infty} \frac{d^{2} g_{i}}{d x^{2}}=0, \quad i=1,2 .
$$

Substituting Eq. (28) into Eq. (27) leads to the following equations for $g_{1}(x)$ and $g_{2}(x)$ :

$$
\left\{\begin{array}{l}
-\beta \frac{d g_{1}}{d x}+\frac{1}{2} \frac{d^{2} g_{1}}{d x^{2}} \\
+\left(-\frac{1}{2} p^{2}+\frac{1}{2} \beta^{2}-2 \mu+3 \mu \operatorname{sech}^{2}(\beta x)-V\right) g_{1}=0 \\
-\beta \frac{d g_{2}}{d x}+\frac{1}{2} \frac{d^{2} g_{2}}{d x^{2}} \\
+\left(-\frac{1}{2} p^{2}+\frac{1}{2} \beta^{2}+\mu \operatorname{sech}^{2}(\beta x)-V\right) g_{2}=0
\end{array}\right.
$$

Considering the asymptotic behavior of Eq. (30) in the limit $|x| \rightarrow \infty$, noting that $V \rightarrow 0$ and $\operatorname{sech}^{2}(\beta x) \rightarrow 0$, and taking Eq. (29) into regard, we obtain

$$
\left\{\begin{array}{l}
\left(-\frac{1}{2} p^{2}+\frac{1}{2} \beta^{2}-2 \mu\right) \tilde{g}_{1}=0, \\
\left(-\frac{1}{2} p^{2}+\frac{1}{2} \beta^{2}\right) \tilde{g}_{2}=0
\end{array}\right.
$$

where $\tilde{g}_{1} \equiv g_{1}(| \pm \infty|)$ and $\tilde{g}_{2} \equiv g_{2}(| \pm \infty|)$ are constants. Equation (31) has, therefore, two cases to discuss.

Case 1: If $\tilde{g}_{2}=0$, then, from the first equation in Eq. (31), we have $p^{2}=\beta^{2}-4 \mu$.However, using Eq. (11) and Eq. (12), this result yields a sign contradiction for small $|A|$. Thus, this case does not yield any sensible solution.

Case 2: If $\tilde{g}_{1}=0$, then, from the second equation in Eq. (31), we have $p^{2}=\beta^{2}$. Therefore, the critical wave number for the instability region of the solution Eq. (14) is given by

$$
k_{\mathrm{cr}}=\beta,
$$

where $\beta$ is determined by Eq. (13). It is interesting to note that the stability threshold does not depend explicitly on the potential barrier; this is a consequence of employing the limit of $x \rightarrow \pm \infty$ in Eq. (30), where the tail of the potential decays to zero. Nonetheless, the effect of the potential barrier is felt, indirectly, by the DSS through the change of its width $\beta$ determined by Eq. (13).
[1] M. H. Anderson, J. R. Ensher, M. R. Matthews, C. E. Wieman, and E. A. Cornell, Science 269, 198 (1995);
K. B. Davis, M.-O. Mewes, M. R. Andrews, N. J. van 
Druten, D. S. Durfee, D. M. Kurn, and W. Ketterle, Phys. Rev. Lett. 75, 3969 (1995); C. C. Bradley, C. A. Sackett, J. J. Tollett, and R. G. Hulet, Phys. Rev. Lett. 75, 1687 (1995).

[2] P. G. Kevrekidis, D. J. Frantzeskakis, and R. CarreteroGonzález (eds). Emergent Nonlinear Phenomena in BoseEinstein Condensates: Theory and Experiment (Springer Series on Atomic, Optical, and Plasma Physics, Vol. 45, 2008).

[3] R. Carretero-González, D. J. Frantzeskakis, and P. G. Kevrekidis, Nonlinearity 21, R139 (2008).

[4] S. Inouye M. R. Andrews, J. Stenger, H.-J. Miesner, D. M. Stamper-Kurn and W. Ketterle, Nature 392, 151 (1998); J. Stenger, S. Inouye, M. R. Andrews, H.-J. Miesner, D. M. Stamper-Kurn, and W. Ketterle, Phys. Rev. Lett. 82, 2422 (1999); J. L. Roberts, N. R. Claussen, J. P. Burke, Jr., C. H. Greene, E. A. Cornell, and C. E. Wieman, Phys. Rev. Lett. 81, 5109 (1998); S. L. Cornish, N. R. Claussen, J. L. Roberts, E. A. Cornell, and C. E. Wieman, Phys. Rev. Lett. 85, 1795 (2000).

[5] F. K. Fatemi, K. M. Jones, and P. D. Lett, Phys. Rev. Lett. 85, 4462 (2000); M. Theis, G. Thalhammer, K. Winkler, M. Hellwig, G. Ruff, R. Grimm, and J. H. Denschlag, Phys. Rev. Lett. 93, 123001 (2004); D. M. Bauer, M. Lettner, C. Vo, G. Rempe, and S. Dürr, Nature Phys. 5, 339 (2009).

[6] E. Haller, M. J. Mark, R. Hart, J. G. Danzl, L. Reichsöllner, V. Melezhik, P. Schmelcher, and H.-C. Nägerl, arXiv:1002.3795.

[7] V. A. Brazhnyi and V. V. Konotop, Mod. Phys. Lett. B 18, 627 (2004); O. Morsch and M. K. Oberthaler, Rev. Mod. Phys. 78, 179 (2006).

[8] K. E. Strecker, G. B. Partridge, A. G. Truscott, and R. G. Hulet, Nature (London) 417, 150 (2002).

[9] L. Khaykovich, F. Schreck, G. Ferrari, T. Bourdel, J. Cubizolles, L. D. Carr, Y. Castin, and C. Salomon, Science 296, 1290 (2002).

[10] S. L. Cornish, S. T. Thompson, and C. E. Wieman, Phys. Rev. Lett. 96, 170401 (2006)

[11] S. Burger, K. Bongs, S. Dettmer, W. Ertmer, K. Sengstock, A. Sanpera, G. V. Shlyapnikov, and M. Lewenstein, Phys. Rev. Lett. 83, 5198 (1999).

[12] J. Denschlag, J. E. Simsarian, D. L. Feder, C. W. Clark, L. A. Collins, J. Cubizolles, L. Deng, E. W. Hagley, K. Helmerson, W. P. Reinhardt, S. L. Rolston, B. I. Schneider, and W. D. Phillips, Science 287, 97 (2000).

[13] C. Becker, S. Stellmer, P. Soltan-Panahi, S. Dörscher, M. Baumert, E.-M. Richter, J. Kronjäger, K. Bongs, and K. Sengstock, Nature Phys. 4, 496 (2008).

[14] S. Stellmer, C. Becker, P. Soltan-Panahi, E.-M. Richter, S. Dörscher, M. Baumert, J. Kronjäger, K. Bongs, and K. Sengstock, Phys. Rev. Lett. 101, 120406 (2008).

[15] A. Weller, J. P. Ronzheimer, C. Gross, J. Esteve, M. K. Oberthaler, D. J. Frantzeskakis, G. Theocharis, and P. G. Kevrekidis, Phys. Rev. Lett. 101, 130401 (2008); G. Theocharis, A. Weller, J.P. Ronzheimer, C. Gross, M.K. Oberthaler, P. G. Kevrekidis, D. J. Frantzeskakis, Phys. Rev. A 81, 063604 (2010).

[16] I. Shomroni, E. Lahoud, S. Levy, and J. Steinhauer, Nature Phys. 5, 193 (2008).

[17] M. A. Hoefer, P. Engels, and J. J. Change, Physica D
238, 1311 (2009)

[18] B. Eiermann, Th. Anker, M. Albiez, M. Taglieber, P. Treutlein, K. P. Marzlin, and M. K. Oberthaler, Phys. Rev. Lett. 92, 230401 (2004).

[19] Yu. S. Kivshar and G. P. Agrawal, Optical Solitons: From Fibers to Photonic Crystals (Academic Press: San Diego, 2003).

[20] B. A. Malomed, D. Mihalache, F. Wise, and L. Torner, J. Opt. B 7, R53 (2005).

[21] Yu. S. Kivshar and B. Luther-Davies, Phys. Rep. 298, $82(1998)$.

[22] D. J. Frantzeskakis, J. Phys. A: Math. Theor. 43, 213001 (2010)

[23] E. A. Kuznetsov and S. K. Turitsyn, Zh. Eksp. Teor. Fiz. 94, 119 (1988) [Sov. Phys. JEPT 67, 1583 (1988)].

[24] E. A. Kuznetsov and J. J. Rasmussen, Phys. Rev. E 51, 4479 (1995).

[25] D. E. Pelinovsky, Yu. A. Stepanyants, and Yu. S. Kivshar, Phys. Rev. E 51, 5016 (1995).

[26] V. Tikhonenko, J. Christou, B. Luther-Davies, and Yu. S. Kivshar, Opt. Lett. 21, 1129 (1996).

[27] A. V. Mamaev, M. Saffman, and A. A. Zozulya, Phys. Rev. Lett. 76, 2262 (1996).

[28] Yu. S. Kivshar and D. E. Pelinovsky, Phys. Rep. 331, 117 (2000).

[29] B. P. Anderson, P. C. Haljan, C. A. Regal, D. L. Feder, L. A. Collins, C. W. Clark, and E. A. Cornell, Phys. Rev. Lett. 86, 2926 (2001).

[30] D. L. Feder, M. S. Pindzola, L. A. Collins, B. I. Schneider, and C. W. Clark, Phys. Rev. A 62, 053606 (2000).

[31] G. A. Swartzlander Jr., D. R. Andersen, J. J. Regan, H. Yin, and A. E. Kaplan, Phys. Rev. Lett. 66, 1583 (1991).

[32] P. G. Kevrekidis, G. Theocharis, D. J. Frantzeskakis, and A. Trombettoni, Phys. Rev. A 70, 023602 (2004).

[33] J. Brand and W. P. Reinhardt, Phys. Rev. A 65, 043612 (2002).

[34] Y. Lin, R.-K. Lee, and Yu. S. Kivshar, J. Opt. Soc. Am. B 25, 576 (2008).

[35] R. Nath, P. Pedri, and L. Santos, Phys. Rev. Lett. 101, 210402 (2008).

[36] T. Lahaye, C. Menotti, L. Santos, M. Lewenstein, and T. Pfau, Rep. Progr. Phys. 72, 126401 (2009).

[37] A. Armaroli, S. Trillo, and A. Fratalocchi, Phys. Rev. A 80, 053803 (2009).

[38] B. A. Malomed, H. E. Nistazakis, D. J. Frantzeskakis, and P. G. Kevrekidis, Phys. Rev. A 70, 043616 (2004).

[39] B. A. Malomed, H. E. Nistazakis, D. J. Frantzeskakis, and P. G. Kevrekidis, Math. Comput. Simul. 69, 400 (2005).

[40] R. Grimm, M. Weidemüller, and Y. B. Ovchinnikov, Adv. At. Mol. Opt. Phys. 42, 95 (2000).

[41] Yu. S. Kivshar and W. Królikowski, Opt. Commun. 114, 353 (1995).

[42] G. Theocharis, P. Schmelcher, M. K. Oberthaler, P. G. Kevrekidis, and D. J. Frantzeskakis, Phys. Rev. A 72, 023609 (2005).

[43] D. J. Frantzeskakis, G. Theocharis, F. K. Diakonos, P. Schmelcher, and Yu. S. Kivshar, Phys. Rev. A 66, 053608 (2002). 